TAIWANESE JOURNAL OF MATHEMATICS

Vol. 3, No. 4, pp. 539-546, December 1999

\title{
A LIMIT PROPERTY OF ARBITRARY DISCRETE INFORMATION SOURCES
}

\author{
Liu Wen
}

\begin{abstract}
In this paper the notion of stochastic conditional entropy is introduced, and the asymptotic relation between this notion and the relative entropy density is studied. A strong limit theorem which holds for arbitrary discrete information sources is obtained. In the proof an analytic technique to study the strong limit properties of discrete information sources is proposed.
\end{abstract}

\section{INTRODUCTION}

A question of importance in information theory is the study on the ShannonMcMillan-Breiman theorem. In previous works, conditions such as ergodicity, stationarity or asymptotic stationarity were assumed (cf. [1]-[2], [4]-[9]). In this paper we avoid these assumptions and give a strong limt theorem concerning relative entropy density and random conditional entropy, which holds for arbitrary discrete information sources. In the proof an analytic technique to study the strong limit properties of discrete information sources is proposed. The crucial part is the application of Lebesgue's theorem on differentiability of monotone functions together with the convergence theorem of infinite products.

Let $\left\{X_{n}, n \geq 1\right\}$ be a sequence of successive letters produced by an arbitrary information source with the alphabet $S=\{1,2, \ldots, N\}$ and with the joint distribution

$$
P\left(X_{1}=x_{1}, \ldots, X_{n}=x_{n}\right)=p\left(x_{1}, \ldots, x_{n}\right)>0 .
$$

Received July 31, 1997; revised December 4, 1998.

Communicated by Y.-J. Lee.

1991 Mathematics Subject Classification: 94A17, 60F15.

Key words and phrases: Random conditional entropy, relative entropy density, strong limit theorem, Shannon-McMillan-Breiman theorem. 
Let

$$
f_{n}(\omega)=-(1 / n) \ln p\left(X_{1}, \ldots, X_{n}\right),
$$

where $\omega$ is a sample point, $X_{i}$ stands for $X_{i}(\omega)$ for brief, and the quantity $f_{n}(\omega)$ is called the relative entropy density of $\left\{X_{i}, 1 \leq i \leq n\right\}$ (see [2]). Let

$$
p_{n}\left(x_{n} \mid x_{1}, \ldots, x_{n-1}\right)=P\left(X_{n}=x_{1}, \ldots, X_{n-1}=x_{n-1}\right), \quad n \geq 2 .
$$

Then

$$
\begin{gathered}
p\left(x_{1}, \ldots, x_{n}\right)=p\left(x_{1}\right) \prod_{k=2}^{n} p_{k}\left(x_{k} \mid x_{1}, \ldots, x_{k-1}\right) \\
f_{n}(\omega)=-(1 / n)\left[\ln p\left(X_{1}\right)+\sum_{k=2}^{n} \ln p_{k}\left(X_{k} \mid X_{1}, \ldots, X_{k-1}\right)\right] .
\end{gathered}
$$

\section{A realization of Arbitrary Information Source}

Throughout this paper we shall deal with the underlying probability space $([0,1), \boldsymbol{F}, P)$, where $\mathrm{F}$ is the class of Borel sets in the interval $[0,1)$, and $P$ is the Lebesgue measure. We first give a realization of an arbitrary information source with distribution (1) in the above probability space.

Split the interval $[0,1)$ into $N$ right-semiopen intervals:

$$
I_{1}=[0, p(1)), \quad I_{2}=[p(1), p(1)+p(2)), \ldots, \quad I_{N}=[1-p(N), 1) .
$$

These intervals will be called intervals of the first order. Proceeding inductively by splitting each $n$th order interval $I_{X_{1} \cdots X_{n}}$ into $N$ right-semiopen intervals $I_{x_{1} \cdots x_{n} 1}, I_{x_{1} \cdots x_{n} 2}, \ldots, I_{x_{1} \cdots x_{n} N}$ according to the ratio $p\left(x_{1}, \ldots, x_{n}, 1\right)$ : $p\left(x_{1}, \ldots, x_{n}, 2\right): \ldots: p\left(x_{1}, \ldots, x_{n}, N\right)$, the intervals of the $(n+1)$ st order are created. It is easy to see that for $n \geq 1$,

$$
P\left(I_{x_{1} \cdots x_{n}}\right)=p\left(x_{1}, \ldots, x_{n}\right) .
$$

Define, for $n \geq 1$, a random variable $X_{n}:[0,1) \rightarrow S$ as follows:

$$
X_{n}(\omega)=x_{n}, \quad \text { if } \omega \in I_{x_{1} \cdots x_{n}} .
$$

By (6) and (7), $\left\{X_{n}, n \geq 1\right\}$ has the distribution (1).

We are going to prove the following limit theorem by using the above realization. 


\section{MAin Result}

Definition 1. For $k \geq 2$, let

(8)

$$
\begin{gathered}
h_{k}\left(x_{1}, \ldots, x_{k-1}\right)=-\sum_{x_{k}=1}^{N} p_{k}\left(x_{k} \mid x_{1}, \ldots, x_{k-1}\right) \ln p_{k}\left(x_{k} \mid x_{1}, \ldots, x_{k-1}\right) ; \\
H_{k}(\omega)=h_{k}\left(X_{1}, \ldots, X_{k-1}\right) .
\end{gathered}
$$

$H_{k}(\omega)$ is called the random conditional entropy.

Theorem 1. Let $\left\{X_{n}, n \geq 1\right\}$ be a sequence of succesive letters produced by an arbitrary information source with the alphabet $S$ and the joint distribution (1), $\left\{a_{n}, n \geq 1\right\}$ an increasing sequence of positive real numbers such that

$$
\sum_{n=1}^{\infty}\left(1 / a_{n}\right)^{2}<\infty
$$

and $f_{n}(\omega)$ and $H_{k}(\omega)$ defined, respectively, by (2) and (9). Then

$$
\begin{aligned}
& \sum_{k=2}^{\infty}\left(1 / a_{k}\right)\left[\ln p_{k}\left(X_{1}, \ldots, X_{k-1}\right)+H_{k}(\omega)\right] \text { converges a.e.; } \\
& \lim _{n \rightarrow \infty}\left(1 / a_{n}\right) \sum_{k=2}^{n}\left[\ln p_{k}\left(X_{k} \mid X_{1}, \ldots, X_{k-1}\right)+H_{k}(\omega)\right]=0 \text { a.e. }
\end{aligned}
$$

Proof. For $k \geq 2, \lambda=1$ or -1 , let

$$
\begin{aligned}
Q_{k}\left(\lambda ; x_{1}, \ldots, x_{k-1}\right)= & \sum_{x_{k}=1}^{N} p_{k}\left(x_{k} \mid x_{1}, \ldots, x_{k-1}\right) \\
& \exp \left\{\lambda \left[\ln p_{k}\left(x_{k} \mid x_{1}, \ldots, x_{k-1}\right)\right.\right. \\
& \left.\left.+h_{k}\left(x_{k} \mid x_{1}, \ldots, x_{k-1}\right)\right] / a_{k}\right\} .
\end{aligned}
$$

Let the collection of intervals of all orders be denoted by $A$. Define a set function $\mu$ on $\mathbf{A}$ as follows. Let

$$
\mu\left(I_{x_{1}}\right)=p\left(x_{1}\right),
$$

and for $n \geq 2$, let 


$$
\begin{aligned}
& \mu\left(I_{x_{1} \cdots x_{n}}\right) \\
& =\frac{p\left(x_{1}, \ldots, x_{n}\right) \exp \left\{\lambda \sum_{k=2}^{n}\left[\ln p_{k}\left(x_{k} \mid x_{1}, \ldots, x_{k-1}\right)+h_{k}\left(x_{1}, \ldots, x_{k-1}\right)\right] / a_{k}\right\}}{\prod_{k=2}^{n} Q_{k}\left(\lambda ; x_{1}, \ldots, x_{k-1}\right)} .
\end{aligned}
$$

By (4) and (13)-(15), it is easy to see that $\mu$ is an additive function on $\mathbf{A}$. Therefore there exists an increasing function $f_{\lambda}$ defined on $[0,1]$ such that, for any $I_{x_{1} \cdots x_{n}}$,

$$
\mu\left(I_{x_{1} \cdots x_{n}}\right)=f_{\lambda}\left(I_{x_{1} \cdots x_{n}}^{+}-f_{\lambda}\left(I_{x_{1} \cdots x_{n}}^{-}\right),\right.
$$

where $I_{x_{1} \cdots x_{n}}^{-}$and $I_{x_{1} \cdots x_{n}}^{+}$denote, respectively, the left and right endpoints of $I_{x_{1} \cdots x_{n}}$. Let

$$
\begin{aligned}
t_{n}(\lambda, \omega) & =\frac{f_{\lambda}\left(I_{x_{1} \cdots x_{n}}^{+}\right)-f_{\lambda}\left(I_{x_{1} \cdots x_{n}}^{-}\right)}{I_{x_{1} \cdots x_{n}}^{+}-I_{x_{1} \cdots x_{n}}^{-}} \\
& =\frac{\mu\left(I_{x_{1} \cdots x_{n}}\right)}{P\left(I_{x_{1} \cdots x_{n}}\right)}, \quad \omega \in I_{x_{1} \cdots x_{n}} .
\end{aligned}
$$

Ler $A(\lambda)$ be the set of points of differentiability of $f_{\lambda}$. Then $P(A(\lambda))=1$ by the theorem on the existence of derivative of monotone function (cf. [3], p. $424)$. Let $\omega \in A(\lambda)$, and $\omega \in I_{x_{1} \cdots x_{n}}(n=1,2, \ldots)$. In virtue of a property of derivative (cf. [3], p. 423), we have by (17),

$$
\lim _{n \rightarrow \infty} t_{n}(\lambda, \omega)=\text { a finite number, } \omega \in A(\lambda) .
$$

By (17), (14), (6) and (7),

$$
\begin{aligned}
t_{n}(\lambda, \omega) & =\frac{\mu\left(I_{X_{1} \cdots X_{n}}\right)}{P\left(I_{X_{1} \cdots X_{n}}\right)} \\
& =\frac{\exp \left\{\lambda \sum_{k=2}^{n}\left[\ln p_{k}\left(X_{k} \mid X_{1}, \ldots, X_{k-1}\right)+h_{k}\left(X_{1}, \ldots, X_{k-1}\right)\right] / a_{k}\right\}}{\prod_{k=2}^{n} Q_{k}\left(\lambda ; X_{1}, \ldots, X_{k-1}\right)} .
\end{aligned}
$$

For the sake of simplicity, denote $p_{k}\left(x_{k} \mid x_{1}, \ldots, x_{k-1}\right)$ and $h_{k}\left(x_{1}, \ldots, x_{k-1}\right)$ by $p_{k}$ and $h_{k}$, respectively. We have by (8),

$$
\sum_{x_{k}=1}^{N} p_{k}\left(\ln p_{k}+h_{k}\right)=0 .
$$


By (13), (20), the inequality $0 \leq e^{x}-1-x \leq x^{2} e^{|x|}$ and the entropy inequality $h_{k} \leq \ln N$, we have

$$
\begin{aligned}
& 0 \leq Q_{k}\left(\lambda ; x_{1}, \ldots, x_{k-1}\right)-1 \\
& =\sum_{x_{k}=1}^{N} p_{k}\left\{\exp \left[\lambda\left(\ln p_{k}+h_{k}\right) / a_{k}\right]-1-\lambda\left(\ln p_{k}+h_{k}\right) / a_{k}\right\} \\
& \leq\left(1 / a_{k}\right)^{2} \sum_{x_{k}=1}^{N} p_{k}\left(\ln p_{k}+h_{k}\right)^{2} \exp \left[\left(-\ln p_{k}+\ln N\right) / a_{k}\right] .
\end{aligned}
$$

Since $a_{k} \rightarrow \infty($ as $k \rightarrow \infty)$, there exists a positive integer $m$ such that $a_{k} \geq 2$ as $k \geq m$. Hence as $k \geq m$, we have by (21) and the entropy inequality,

$$
\begin{aligned}
& 0 \leq Q_{k}\left(\lambda ; x_{1}, \ldots, x_{k-1}\right)-1 \\
& \leq N\left(1 / a_{k}\right)^{2} \sum_{x_{k}=1}^{N} p_{k}^{1 / 2}\left(\ln p_{k}+h_{k}\right)^{2} \\
& <N\left(1 / a_{k}\right)^{2} \sum_{x_{k}=1}^{N}\left[p_{k}^{1 / 2}\left(\ln p_{k}\right)^{2}-2(\ln N) p_{k}^{1 / 2} \ln p_{k}+(\ln N)^{2}\right] .
\end{aligned}
$$

Let

$$
\begin{aligned}
& M_{1}=\max \left\{x^{1 / 2}(\ln x)^{2}, \quad 0<x \leq 1\right\} \\
& M_{2}=\max \left\{-x^{1 / 2} \ln x, \quad 0<x \leq 1\right\} .
\end{aligned}
$$

From (22) and (10) it follows that

$$
\begin{aligned}
& \sum_{k=m}^{\infty}\left[Q_{k}\left(\lambda ; X_{1}, \ldots, X_{k-1}\right)-1\right] \\
& <\sum_{k=m}^{\infty}\left(N / a_{k}\right)^{2}\left\{M_{1}+2 M_{2} \ln N+(\ln N)^{2}\right\}<\infty .
\end{aligned}
$$

By the convergence theorem of infinite product, (23) implies that

$$
\prod_{k=2}^{\infty}\left[Q_{k}\left(\lambda ; X_{1}, \ldots, X_{k-1}\right)\right. \text { converges. }
$$

(18), (19) and (24) imply that

$$
\begin{aligned}
& \lim _{n \rightarrow \infty} \exp \left\{\lambda \sum_{k=2}^{n}\left[\ln p_{k}\left(X_{k} \mid X_{1}, \ldots, X_{k-1}\right)+h_{k}\left(X_{1}, \ldots, X_{k-1}\right)\right] / a_{k}\right\} \\
& =\text { a finite number a.e.. }
\end{aligned}
$$

Letting $\lambda=1$ and $\lambda=-1$ respectively, we have 


$$
\lim _{n \rightarrow \infty} \exp \left\{\sum_{k=2}^{n}\left[\ln p_{k}\left(X_{k} \mid X_{1}, \ldots, X_{k-1}\right)+h_{k}\left(X_{1}, \ldots, X_{k-1}\right)\right] / a_{k}\right\}
$$

$=\mathrm{a}$ finite number a.e.

$$
\begin{aligned}
& \lim _{n \rightarrow \infty} \exp \left\{-\sum_{k=2}^{n}\left[\ln p_{k}\left(X_{k} \mid X_{1}, \ldots, X_{k-1}\right)+h_{k}\left(X_{1}, \ldots, X_{k-1}\right)\right] / a_{k}\right\} \\
& =\text { a finite number a.e.. }
\end{aligned}
$$

(26) and (27) imply that

(28) $\sum_{k=2}^{n}\left\{\left[\ln p_{k}\left(X_{k} \mid X_{1}, \ldots, X_{k-1}\right)+h_{k}\left(X_{1}, \ldots, X_{k-1}\right)\right] / a_{k}\right\}$ converges a.e.

i. e., (11) is true. By Kronecker's lemma, (12) follows from (28).

This completes the proof of the theorem.

\section{Some Corollaries}

Corollary 1. Let $f_{n}(\omega)$ be defined by (5). Then under the hypotheses of the theorem we have

$$
\lim _{n \rightarrow \infty}\left[f_{n}(\omega)-(1 / n) \sum_{k=1}^{n} H_{k}(\omega)\right]=0 \text { a.e.. }
$$

Proof. Let $a_{n}=1 / n$. Then (29) follows from (12) and (5) immediately.

Corollary 2. Let $p>1 / 2$ be a constant. Then under the hypotheses of the theorem we have

$$
\lim _{n \rightarrow \infty} n^{-1 / 2}(\ln n)^{-p} \sum_{k=2}^{n}\left[\ln p_{k}\left(X_{k} \mid X_{1}, \ldots, X_{k-1}\right)+H_{k}(\omega)\right]=0 \quad \text { a.e.. }
$$

Proof. Since $\sum_{n=2}^{\infty} n^{-1}(\ln n)^{-2 p}<\infty$, the corollary is obtained immediately from the theorem.

Corollary 3. Let $\left\{X_{n}, n \geq 1\right\}$ be a sequence of successive letters produced by a nonhomogeneous Markov information source with the initial distribution 


$$
p(1), P(2), \cdots, p(N), \quad p(i)>0, \quad i \in S
$$

and the transition matrix

$$
P_{n}=\left(p_{n}(i, j)\right), \quad p_{n}(i, j)>0, \quad i, j \in S, \quad n \geq 1,
$$

where $p_{n}(i, j)=P\left(X_{n}=j \mid X_{n-1}=i\right),\left\{a_{n}, n \geq 1\right\}$ is an increasing sequence of positive numbers such that (10) holds, and $H\left(p_{1}, \ldots, p_{n}\right)$ is the entropy of the distribution $\left(p_{1}, \ldots, p_{n}\right)$. Then

$$
\begin{aligned}
& \lim _{n \rightarrow \infty}\left(1 / a_{n}\right) \sum_{k=1}^{n}\left\{\ln p_{k}\left(X_{k-1}, X_{k}\right)+H\left[p_{k}\left(X_{k-1}, \ldots, p_{k}\left(X_{k-1}, N\right)\right]\right\}\right. \\
& =0 \quad \text { a.e.. }
\end{aligned}
$$

Proof. By Markov property, (33) follows from (12) immediately.

Corollary 4. Let $p>1 / 2$ be a constant. Then under the hypotheses of Corollary 3 we have

$$
\lim _{n \rightarrow \infty}\left\{f_{n}(\omega)-(1 / n) \sum_{k=1}^{n} H\left[p_{k}\left(X_{k-1}, 1\right), \ldots, p_{k}\left(X_{k-1}, N\right)\right]\right\}=0 \quad \text { a.e. }
$$

where

$$
f_{n}(\omega)=-(1 / n)\left[\ln p\left(X_{0}\right)+\sum_{k=1}^{n} \ln p_{k}\left(X_{k-1}, X_{k}\right)\right]
$$

is the relative entropy density of the Markov information source.

Proof. Let $a_{n}=n$. Then (34) follows from (33) and (5) directly.

\section{REFERENCES}

1. P. H. Algoet and T. M. Cover, A sandwich proof of the Shannon-McMillanBreiman theorem, Ann. Probab. 16 (1988), 899-909.

2. A. R. Barron, The strong ergodic theorem for densities: Generalized ShannonMcMillan-Breiman theorem, Ann. Probab. 13 (1985), 1292-1303.

3. P. Billingsley, Probability and Measure, Wiley, New York, 1986.

4. L. Breiman, The individual ergodic theorem of information theory, Ann. Math. Statist. 28 (1957), 809-811. 
5. K. L. Chung, The ergodic theorem of information theory, Ann. Math. Statist. 32 (1961), 612-614.

6. J. C. Kieffer, A counterexample to Perez's generalization of the ShannonMcMillan theorem, Ann. Probab. 1 (1973), 362-364.

7. J. C. Kieffer, A counterexample to Perez's generalization of the ShannonMcMillan theorem, Pacific J. Math. 51 (1973), 203-204.

8. B. McMillan, The basic theorems of information theory, Ann. Math. Statist. 24 (1953), 196-216.

9. C. Shannon, A mathematial theory of communication, Bell System Tech. J. 27 (1948), 379-423, 623-656.

Department of Mathematics, Hebei University of Technology

Tianjin 300130, China 